\title{
EGFR C797S as a Resistance Mechanism of Lazertinib in Non-small Cell Lung Cancer with EGFR T790M Mutation
}

\author{
Sehhoon Park, MD \\ Bo Mi Ku, PhD² \\ Hyun Ae Jung, MD, PhD' \\ Jong-Mu Sun, MD, PhD' \\ Jin Seok Ahn, MD, PhD' \\ Se-Hoon Lee, MD, PhD' \\ Keunchil Park, MD, PhD' \\ Myung-Ju Ahn, MD, PhD ${ }^{1}$
}

The non-small cell lung cancer with activating epidermal growth factor receptor (EGFR) mutation eventually acquires resistant to either first or second-generation EGFR tyrosine kinase inhibitor (TKI). As the following option, targeting EGFR T790M with third-generation EGFR TKI is now established as a standard treatment option. In this study, we are reporting the first case of resistance mechanism to the novel third-generation EGFR TKI, lazertinib, which showed promising clinical efficacy in phase 1-2 study. The patients showed resistance to the treatment by acquiring the additional EGFR C797S mutation in cis which is also confirmed from the patient-derived cell lines.

\section{Key words}

Non-small cell lung cancer, Lazertinib, Third-generation EGFR tyrosine kinase inhibitor, ErbB receptors

Correspondence: Myung-Ju Ahn, MD, PhD

Division of Hematology-Oncology,

Department of Medicine, Samsung Medical

Center, Sungkyunkwan University School of

Medicine, 81 Irwon-ro, Gangnam-gu,

Seoul 06351, Korea

Tel: 82-2-3410-3438

Fax: 82-2-3410-1754

Email: silkahn@skku.edu

Received April 5, 2020

Accepted June 19, 2020

Published Online June 22, 2020

\section{Introduction}

Non-small cell lung cancer (NSCLC) with activating epidermal growth factor receptor (EGFR) mutation treated with either a first- or second-generation tyrosine kinase inhibitor (TKI) can experience treatment failure, most commonly by acquiring an additional genomic alteration in EGFR T790M [1]. Lazertinib (YH25448) is a potent irreversible third-generation EGFR TKI that targets both T790M and activating EGFR mutation with high penetration to the blood-brain barrier. Lazertinib showed promising anti-tumor efficacy with a 57\% overall response rate and 9.7-month median progression-free survival in EGFR T790M-positive patients [2]. However, there is no previous report showing the mechanism of tumor resist- ance acquisition to lazertinib. In this case report, we conducted deep-targeted sequencing of resistant tumor samples and established patient-derived cell lines (PDC) from a patient treated with lazertinib to elucidate the underlying genomic alteration associated with resistance.

\section{Case Report}

A 38-year-old current male smoker presented with stage 4, cT1bN3M1b, NSCLC adenocarcinoma. Informed consent was received under supervision of institutional review board (SMC 2011-10-054-034). The patient was shown to harbor an EGFR exon 19 deletion using real-time polymerase chain 
A

Mean depth
SNV/INDEL

\begin{tabular}{c|c} 
& Del. 19 \\
\cline { 2 - 2 } EGFR & T790M \\
\cline { 2 - 2 } & C797S \\
\hline & \\
\hline PTCH1 & D1305Y \\
\hline ABL2 & P878R \\
\hline BCR & A1149T \\
\hline ERCC2 & H148R \\
\hline FANCD2 & 065H \\
\hline FANCG & R3L \\
\hline FGF10 & M204V \\
\hline FGFR2 & R6P \\
\hline LRP1B & R45730 \\
\hline PKHD1 & W1725S \\
\hline TSC1 & S487C \\
\hline GNA0 & \\
EP300 &
\end{tabular}

Fusion

WYDYHV1

-CRLF2
B
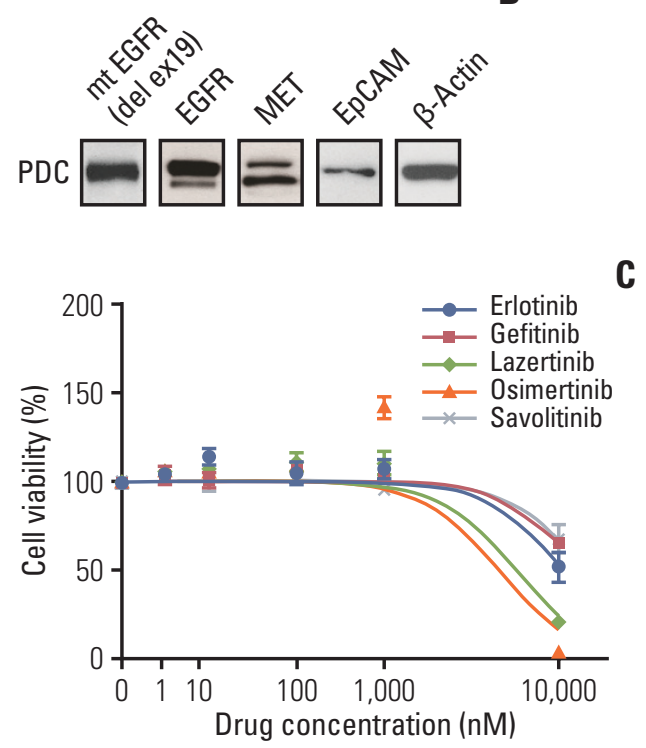

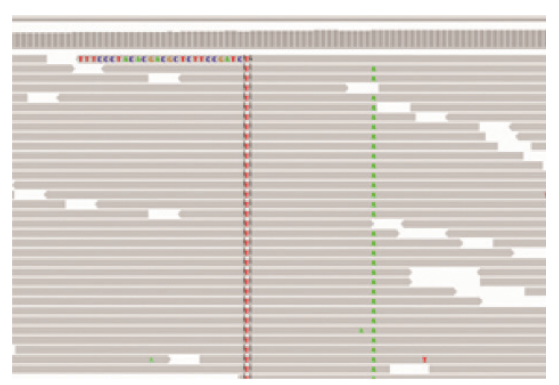

Fig. 1. (A) Target sequencing in samples from lazertinib-resistant malignant ascites. Integrative genomic viewer of sample showing additional epidermal growth factor receptor (EGFR) C797S cis mutation. (B) Western blot of patient-derived cell line (PDC) samples. (C) Cell viability analyses conducted in samples after exposure to tyrosine kinase inhibitor for 72 hours. SNV, single nucleotide variant; INDEL, insertion/deletion; EpCAM, epithelial cell adhesion molecule.

reaction from the initial biopsy sample obtained from the mediastinal lymph node. Afatinib was administered as a firstline treatment and showed very good partial response with 7.1 months of progression-free survival. After first-line EGFR TKI failure, a second biopsy from the newly progressed metastatic lymph node showed acquired EGFR T790M mutation.
As a subsequent treatment, the patient received lazertinib as a part of a clinical trial (NCT03046992, YH25448-201). However, after 6.2 months of partial response to lazertinib, the patient developed malignant ascites, suggesting peritoneal seeding due to resistance.

Deep-targeted sequencing (CancerSCAN [3]) of ascites 
samples demonstrated acquired EGFR C797S mutation in cis, variant allele frequency (VAF) of 9.4\%, EGFR T790M (VAF of $3.5 \%$ ), and EGFR exon 19 deletion (VAF 9.2\%) (Fig. 1A). PDCs established from the same ascites sample showed EGFR exon 19 deletion (Fig. 1B). Cell viability of PDCs showed resistance to first- and third-generation EGFR TKIs including erlotinib, gefitinib, lazertinib, and osimertinib or the c-Met inhibitor savolitinib (Fig. 1C).

\section{Discussion}

Diverse resistance mechanisms to third-generation EGFR TKI have been reported including loss of T790M, acquisition of EGFR C797S mutation, c-Met amplification, activation of other bypass tract, or small cell lung cancer transformation. Among them, EGFR C797S/T790M mutation is the most frequently observed, accounting for $20 \%-30 \%$ of cases. It is of note that tumors acquiring an additional mutation of EGFR C797X maintain the original EGFR T790M mutation [4]. In this report, we present the first clinical case of new EGFR C797S/T790M mutation in a patient who failed lazertinib. Further validation with a large number of patients and new treatment strategies to overcome this resistance mutation are warranted.

\section{Conflicts of Interest}

Conflict of interest relevant to this article was not reported.

\section{References}

1. Sequist LV, Waltman BA, Dias-Santagata D, Digumarthy S, Turke AB, Fidias P, et al. Genotypic and histological evolution of lung cancers acquiring resistance to EGFR inhibitors. Sci Transl Med. 2011;3:75ra26.

2. Ahn MJ, Han JY, Lee KH, Kim SW, Kim DW, Lee YG, et al. Lazertinib in patients with EGFR mutation-positive advanced non-small-cell lung cancer: results from the dose escalation and dose expansion parts of a first-in-human, open-label, multicentre, phase 1-2 study. Lancet Oncol. 2019;20:1681-90.
3. Shin HT, Choi YL, Yun JW, Kim NK, Kim SY, Jeon HJ, et al. Prevalence and detection of low-allele-fraction variants in clinical cancer samples. Nat Commun. 2017;8:1377.

4. Oxnard GR, Hu Y, Mileham KF, Husain H, Costa DB, Tracy $\mathrm{P}$, et al. Assessment of resistance mechanisms and clinical implications in patients with EGFR T790M-positive lung cancer and acquired resistance to osimertinib. JAMA Oncol. 2018;4:1527-34. 